\title{
A contribuição da Engenharia Urbana na solução de problemas territoriais ${ }^{1}$
}

\section{The contribution of Urban Engineering in the solution of territorial problems}

\section{Fritz, Rodrigo Tenório'; Pfeiffer, Cláudia Ribeiro2; Pina Filho, Armando Carlos ${ }^{3}$}

\begin{abstract}
I Universidade Federal do Rio de Janeiro, Programa de Engenharia Urbana, Escola Politécnica, Av. Athos da Silveira Ramos, 149, Cidade Universitária, 21941-485, Rio de Janeiro - RJ, Brasil, rffritz@poli.ufrj.br

2 Universidade Federal do Rio de Janeiro, Programa de Engenharia Urbana, claudia.pfeiffer@poli.ufrj.br

3 Universidade Federal do Rio de Janeiro, Programa de Engenharia Urbana, armando@poli.ufrj.br
\end{abstract}

\section{RESUMO}

A Engenharia Urbana desempenha um papel fundamental no planejamento e gestão territorial, orientando a alocação de recursos de forma correta, auxiliando na adequada tomada de decisões, mobilizando todos os setores para priorizar o pensamento coletivo, criando planos de metas para o desenvolvimento das cidades e agenda de ações de forma participativa. Dessa forma, o presente trabalho busca apresentar uma visão geral dos conceitos de engenharia urbana, planejamento e gestão territorial, visando observar a contribuição da engenharia urbana na solução de problemas territoriais. A metodologia consiste numa revisão bibliográfica e, como resultado, espera-se apresentar a engenharia urbana como uma ciência que agrega o conhecimento das engenharias setoriais com o planejamento e gestão territorial. Nesse sentido, destacam-se ações importantes na definição de metas e prazos para tomada de decisões, baseando-se na participação popular para ajustar os problemas e soluções. Então, definindo de que forma a contribuição da engenharia é importante para solucionar e priorizar esses problemas urbanos.

Palavras-chave: Engenharia Urbana, Planejamento e Gestão Territorial, Ações Participativas.

\begin{abstract}
Urban Engineering plays a fundamental role in territorial planning and management, guiding the allocation of resources in a correct way, assisting in proper decision making, mobilizing all sectors to prioritize collective thinking, creating plans of goals for the development of cities, and agenda of actions in a participative way. Thus, the present work search to show an overview of the concepts of urban engineering, territorial planning and management, aiming to observe the contribution of urban engineering in the solution of territorial problems. The

\footnotetext{
${ }^{1}$ FRITZ, Rodrigo Tenório; PFEIFFER, Cláudia Ribeiro; PINA FILHO, Armando Carlos. A contribuição da Engenharia Urbana na solução de problemas territoriais. In: II SIMPÓSIO NACIONAL DE GESTÃO E ENGENHARIA URBANA: SINGEURB, 2019, São Paulo. Anais... Porto Alegre: ANTAC, 2019.
} 
methodology consists of a bibliographical review and, as a result, it is expected to present urban engineering as a science that aggregates the knowledge of sectorial engineering with territorial planning and management. In this sense, important actions are highlighted in setting goals and deadlines for decision making, based on popular participation to adjust problems and solutions. Then, defining how the contribution of engineering is important to solve and prioritize these urban problems.

Keywords: Urban Engineering, Territorial Planning and Management, Participatory Actions.

\section{INTRODUÇÃO}

A Engenharia Urbana tem papel fundamental no auxílio ao planejamento e gestão territorial. Sua importância se destaca pela orientação na alocação de recursos de forma correta, auxiliando na tomada de decisões de maneira assertiva, na mobilização de todos os setores para priorizar o pensamento coletivo, criando planos de metas para o desenvolvimento das cidades, bem como pauta de ações de forma participativa, com a unificação de atores para resolução de problemas do território em um ambiente colaborativo. Um interessante trabalho sobre a conceituação e desafios da Engenharia Urbana foi publicado por Abiko (2010) e, mais recentemente, Silva (2018) realizou um estudo das contribuições da Engenharia Urbana para o desenvolvimento urbano sustentável.

Com relação à governança, é um princípio fundamental para se planejar uma cidade sustentável, estabelecendo as diretrizes, as metas e as responsabilidades de cada setor, público ou privado, reforçado pelo envolvimento da sociedade, possibilitando implementar uma gestão eficiente que envolva as etapas de planejamento, execução e avaliação. Bento (2003) apresenta um estudo interessante sobre governança e governabilidade, enquanto Brasil (2014) fornece um referencial básico de governança aplicável a órgãos e entidades da administração pública e ações indutoras de melhoria.

Segundo Maximiano (1995), planejar consiste em tomar decisões antecipadamente. Certas decisões são tomadas de imediato, assim que o problema ocorre, e seu alcance esgota-se com a resolução desse mesmo problema. Outras decisões, ao contrário, visam definir um objetivo ou curso de ação para o futuro, de curto a longo prazo.

Dessa forma, com o planejamento e gestão urbana é possível reduzir problemas enfrentados pela cidade, direcionando as ações que devem ser tomadas para mitigar os problemas e minimizar os efeitos que eles causam. Moscarelli e Kleiman (2017) apresentam um estudo recente sobre os desafios do planejamento e gestão urbana integrada no Brasil.

Quanto ao território, no seu sentido geográfico, contém as obras e atividades que nos interessam. É o conjunto de elementos físicos e não-físicos que compõem o espaço que nos rodeia na superfície do nosso planeta, que podem incluir desde aspectos topográficos até os de legislação de zoneamento. Vivemos principalmente em cidades, e tal espaço é constituído de seres humanos, que coexistem em densidades variadas, e desenvolvem entre si complexos relacionamentos sociais. Eles se organizam para produzir bens, o que cria complexos relacionamentos econômicos. Para criar os bens, operá-los e mantê-los, são desenvolvidas atividades relacionadas à engenharia (ZMITROWICZ, 2002).

A partir desses conceitos, o presente trabalho busca apresentar uma visão geral dos temas citados, visando observar a contribuição da engenharia urbana na solução de problemas territoriais. A metodologia consiste numa revisão bibliográfica e, como resultado, espera-se apresentar a engenharia urbana como uma ciência que agrega o conhecimento das engenharias setoriais com o planejamento e gestão territorial, como visto na Figura 1.

\section{ENGENHARIA URBANA}

Segundo Pina Filho e Rossi (2013), a engenharia urbana é a área de engenharia que estuda o planejamento, a construção e a gestão territorial, por meio da formulação, da implementação e do gerenciamento de políticas urbanas, além da integração dos diversos 
sistemas urbanos. Para isso, utilizam-se métodos e técnicas específicos, para monitoramento e prospecção de dados, capazes de fornecer ao engenheiro uma visão ampla e sistêmica sobre os diversos fatores sociais, econômicos, políticos, ambientais e tecnológicos, para solução de problemas urbanos, tais como: déficit habitacional, problemas de infraestrutura, serviços ineficientes, poluição ambiental etc., buscando, dessa forma, um desenvolvimento sustentável.

Figura 1 - O conceito de Engenharia Urbana.

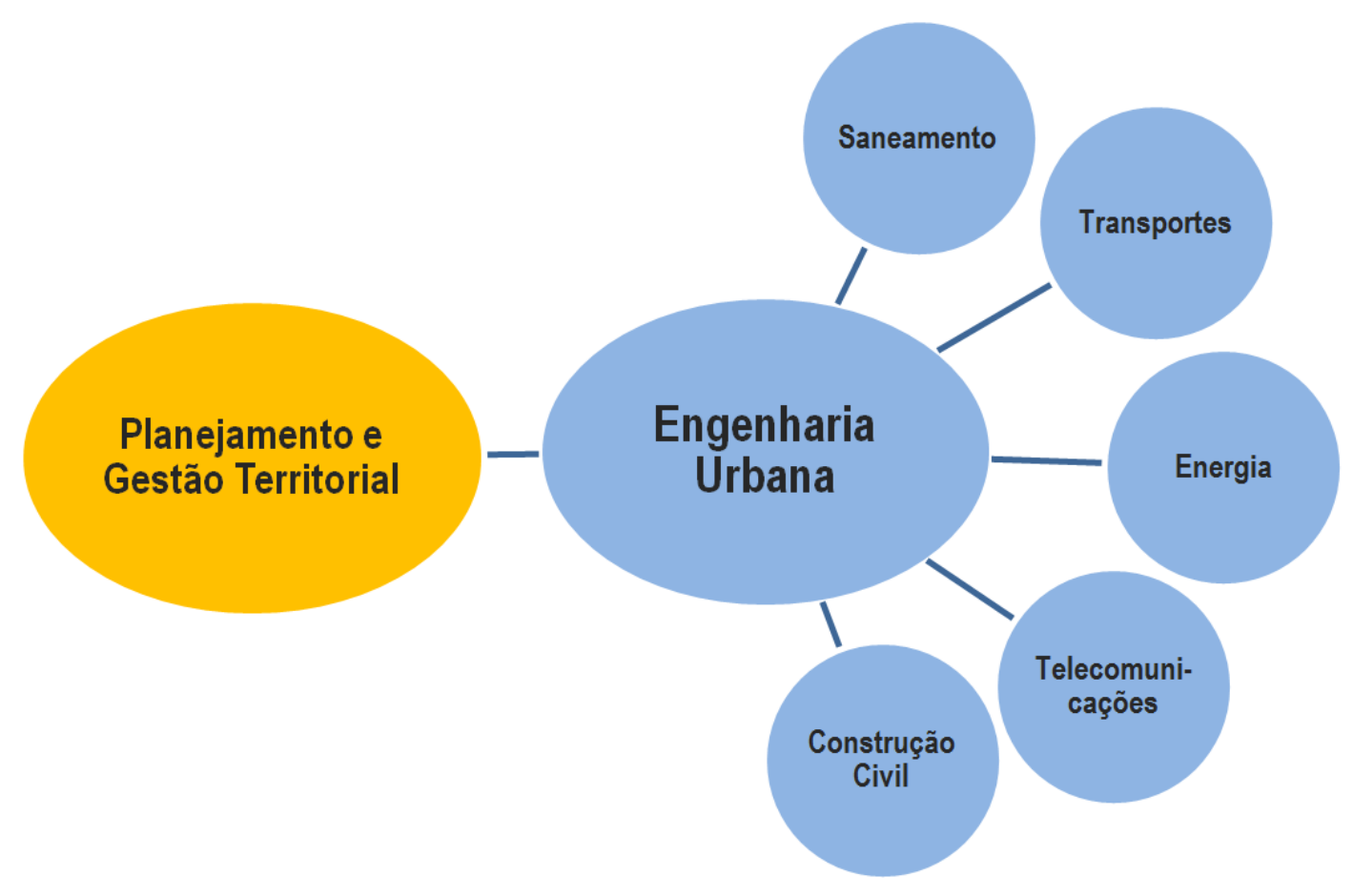

Fonte: Os autores

A engenharia urbana promove a releitura de teorias e conceitos sob a ótica do engenheiro, de forma a aplicar conhecimento técnico para apoio à condução de processos participativos e à tomada de decisões. Não se trata, no entanto, de prevalecer a abordagem da engenharia, mas contribuir para agregá-la ao vasto conjunto do saber já sedimentado pelas demais áreas. Essa integração é de fundamental importância para possibilitar a implementação de soluções para os problemas urbanos, os quais não podem ser analisados isoladamente, mas sim compreendidos sistematicamente, num contexto urbano mais abrangente.

\section{PLANEJAMENTO E GESTÃO TERRITORIAL}

Vitte (2015) nota que o fundamento do planejamento territorial é a gestão dos mais diversos recursos. Entre os constrangimentos das políticas territoriais, aponta-se que não se pode dizer que existe uma política de desenvolvimento econômico territorial no Brasil ou planejamento territorial, mas sim opções políticas que afetam o território e há, muitas vezes, ausência de uma avaliação democrática transparente em relação às demandas sociais nas iniciativas vigentes.

O planejamento e gestão territorial atuam no desenvolvimento das cidades com estruturas e benefícios para a população. A falta de planejamento no Brasil trouxe o crescimento das cidades de forma desordenada, muitas vezes acrescida de problemas de falta de saneamento e infraestrutura. Logo, não se deve satisfazer-se com um único plano, mas com vários planos, tantas quantas forem as previsões do futuro com probabilidade significativa (HUERTAS, 1996). Com isso, o planejamento urbano deve atuar de forma a atenuar os 
problemas que serão enfrentados pelas cidades.

Considerando a cidade como um sistema complexo, ela está sujeita a diversas influências e decisões por diferentes pontos de vistas: sociais, econômicos, políticos, populares e administrativos. Vale ressaltar, que atualmente, no processo de planejamento, também são inseridas novas questões, como a sustentabilidade ambiental, resiliência e a participação social (NOGUEIRA, 2015).

\subsection{Tomada de Decisões}

A engenharia Urbana atua para facilitar as tomadas de decisões que deverão ser escolhidas pela cidade. O ajuste dessas decisões, de forma assertiva, contribui para o melhor desenvolvimento, melhor alocação dos recursos e investimento nos setores mais carentes.

É importante atuar com planejamento para que os recursos sejam alocados de forma correta, assim evitando o desperdício, ou investimentos em locais que não são de necessidade. A engenharia urbana fornece fundamentação técnica para o processo de tomada de decisão. Assim os diversos setores das cidades podem balizar as tomadas de decisões baseadas em uma visão sistêmica.

Moraes (2012) apresentou a influência do fator político na tomada de decisão para projetos de transportes. Esses fatores também se replicam para outros projetos relacionados às cidades. Alguns aspectos são considerados, como posição política, relacionamento dos agentes envolvidos, as ações e recursos que possibilitam o convencimento e o interesse no projeto.

Além disso, de acordo com Rodrigues e Shimoishi (2014), pode-se considerar como fatores de influência em processos decisórios para seleção de tecnologias de transporte: os aspectos econômicos da cidade e dos usuários, aspectos ambientais e políticos, que interferem diretamente ou indiretamente em qualquer decisão relacionados a projetos.

\subsection{Plano de Metas}

O Plano de Metas é um instrumento de planejamento e gestão que é utilizado pela administração pública para definir as prioridades de governo, as metas, prazos e compromissos do setor público.

É importante no desenvolvimento das cidades estabelecer metas e prazos concretos para os projetos que serão desenvolvidos. Ele funciona como um pacto entre os setores públicos, privados e a população, para definir as metas e objetivos concretos a serem cumpridos pelo governo local.

O Plano de Metas é o plano técnico, criado com o auxílio da população, para definir o atual estágio das cidades e estabelecer as metas e prazos que deverão ser atingidas ao longo do período de governo. Assim, a cidade idealizada vai sendo construída a partir desse plano de ações global.

Existem diversos trabalhos sobre plano de metas, sendo o mais notório aquele implementado durante a gestão do então presidente Juscelino Kubitscheck (1956-1961), citado na pesquisa de Cardoso Jr. (2011). Coincidentemente, desde 2011, tramita no Congresso uma Proposta de Emenda à Constituição (PEC 10/2011) que estabelece a obrigatoriedade de elaboração e cumprimento do plano de metas pelo Poder Executivo municipal, estadual e federal, com base nas propostas das campanhas eleitorais. Porém esse item esta paralisado desde 2015 para ser apreciado pelos deputados.

\subsection{Ações Participativas}

A participação da população é de extrema importância na solução de problemas territoriais. A engenharia urbana atua nesse cenário como um mediador técnico das demandas da população com as ações que devem ser tomadas pelo poder público ou privado. Isso pode 
ser visto em trabalhos como o de Araújo (2014).

A ałuação participativa da população é muito importante na definição dos problemas, assim como na priorização das demandas. Os problemas do território podem ser definidos sob a ótica da população, e serem priorizados de acordo com as reivindicações populares.

A participação popular depende da vontade e engajamento de cada cidadão. A legitimidade da participação provém de atitudes distintas: alguns cidadãos agem em prol da participação, por isso reservam tempo e energia para se encorajar, enquanto a maioria não considera necessário investir tempo nesse assunto porque, afinal, não há valorização dessa participação voluntária (GAGLIETTI, 2003). Essa concepção dificulta a ampla participação popular, por isso alguns setores são importantes nesse processo.

Segundo Vieira et al. (2013), existe a importância das instituições de ensino na construção desse processo de participação popular, pois garante legitimidade ao processo, impedindo que somente o Estado tenha poder de decisão. As instituições de Ensino Superior podem oferecer o suporte técnico que gera discussão e melhor entendimento da realidade local, provocando a crítica, o envolvimento e a ampla comunicação política.

Através da participação popular, os problemas territoriais encontrados na cidade podem ser elencados e suas demandas priorizadas. Auxiliado pelos setores técnicos, como a engenharia urbana, pode-se priorizar os maiores problemas e assim atender a população da melhor forma possível. É importante nesse contexto alinhar os benefícios da engenharia urbana como uma ferramenta para gerir e encaminhar as soluções dos problemas. Recomenda-se como consulta o trabalho de Silva e Pfeiffer (2017).

\section{CONSIDERAÇÕES FINAIS}

Com a tendência crescente de urbanização no Brasil e no Mundo, diversos problemas territoriais surgem, principalmente devido à falta de planejamento. Com isso a discussão sobre cidades deve guiar o modelo de planejamento, desenvolvimento urbano inclusivo, solidário e que resgate a relação entre cidadãos e seu território e recursos naturais. A engenharia urbana desempenha papel fundamental nesse cenário, com participação direta no planejamento e gestão, nas tomadas de decisões e priorização das demandas. Isso se dá no pilar ambiental, urbano e social, com políticas de revitalização das cidades, de moradia, de transporte público com qualidade, além de projetos que potencializem o uso eficiente dos recursos naturais no território. Dessa forma, a engenharia auxilia na aplicação de melhores técnicas nos projetos para as cidades, com desenvolvimento tecnológico e inovação para promover um ambiente com desenvolvimento sustentável com participação popular, buscando uma melhor qualidade de vida para população.

\section{REFERÊNCIAS}

$A B I K O, A$. Urban engineering: concepts and challenges. In: Methods and techniques in urban engineering, Ed. Armando Carlos de Pina Filho e Aloísio Carlos de Pina. Viena, Áustria: INTECH Education and Publishing, 2010, p. 1-12. DOI: 10.5772/9570.

ARAÚJO, A. A. Participação da sociedade na decisão urbana. Dissertação de Mestrado. Universidade Federal do Rio de Janeiro, Escola Politécnica, Programa de Engenharia Urbana, Rio de Janeiro - RJ, 2014. 166 p.

\section{BENTO, L. V. Governança e governabilidade na reforma do Estado: entre eficiência e democratização. Barveri: Manole, 2003.}

BRASIL. Tribunal de Contas da União. Governança Pública: referencial básico de governança aplicável a órgãos e entidades da administração pública e ações indutoras de melhoria. Secretaria de Planejamento, Governança e Gestão, Brasília - DF, 2014. 96 p. 
CARDOSO Jr., J. C. Planejamento Governamental e Gestão Pública no Brasil: Elementos para Ressignificar o Debate e Capacitar o Estado. Instituto de Pesquisa Econômica Aplicada (IPEA), Brasília - DF, 2011.

GAGLIETTI, M. J. PT: Ambivalências de uma Militância. Porto Alegre: DACASA, 2003.

HUERTAS, F. O método PES: entrevista com Matus. São Paulo: FUNDAP, 1996.

MAXIMIANO, A. C. A. Introdução à Administração. São Paulo: ATLAS, 1995.

MORAES, A. C. Projetos de Infraestrutura de Transportes: Inserção efetiva na Agenda Governamental. Tese de Doutorado. Programa de Pós-Graduação em Transportes da Universidade de Brasília, 2012.

MOSCARELLI, F.; KLEIMAN, M. Os desafios do planejamento e gestão urbana integrada no Brasil: análise da experiência do Ministério das Cidades. Revista Brasileira de Gestão Urbana, urbe (Brazilian Journal of Urban Management), v. 9, n. 2, p. 157-171, mai./ago. 2017.

NOGUEIRA, A. C. R. M. Planejamento e Gestão Territorial: Uma Análise Sobre as Estratégias de Desenvolvimento Urbano e Ambiental do Município de Maricá/RJ. Trabalho de Conclusão de Curso (Especialização em Engenharia Urbana). Universidade Federal do Rio de Janeiro, Escola Politécnica, Rio de Janeiro, 2015.

PINA FILHO, A. C.; ROSSI, A. M. G. Engenharia Urbana. Revista Brasileira de Gestão Urbana, urbe (Brazilian Journal of Urban Management), v. 5, n. 2, p. 11-12, jul./dez. 2013.

RODRIGUES, S. G.; SHIMOISHI, J. M. Aplicação do Método Paraconsistente de Decisão na Seleção de Tecnologias de Transporte Público Urbano. Journal of Transport Literature, v. 9, n. 3, p. 20-24, July/Sept. 2015.

SILVA, A. A. Contribuições da Engenharia Urbana para o Desenvolvimento Urbano Sustentável: Promovendo a Participação Cívica. Dissertação de Mestrado. Universidade Federal do Rio de Janeiro, Escola Politécnica, Programa de Engenharia Urbana, Rio de Janeiro - RJ, 2018. 121 p.

SILVA, A. A.; PFEIFFER, C. R. Contribuições da Engenharia Urbana para o Desenvolvimento Social e Econômico de uma Localidade: Resgatando a participação cívica e auxiliando processos de governança. In: I Simpósio Nacional de Gestão e Engenharia Urbana, Anais, p. 432-438. São Carlos: Universidade Federal de São Carlos, 2017.

VIEIRA, R.; PEREIRA, L. N.; DOS ANJOS, F. A.; SCHROEDER, T. Participação popular no processo de planejamento urbano: a universidade como "decodificadora" de um sistema de muitos códigos. Revista Brasileira de Gestão Urbana, urbe (Brazilian Journal of Urban Management), v. 5, n. 2, p. 115-130, jul./dez. 2013.

VITTE, C. C. S. O planejamento territorial e a dimensão espacial do desenvolvimento: algumas das experiências recentes no Brasil. Revista Política e Planejamento Regional, v. 2, n. 1, p. 1-18, jan./junho 2015.

ZMITROWICZ, W. Planejamento Territorial Urbano. Texto Técnico, Escola Politécnica, Universidade de São Paulo, São Paulo, 2002. 